\title{
A double-negative feedback loop between EZH2 and miR-26a regulates tumor cell growth in hepatocellular carcinoma
}

\author{
CHUNBO ZHUANG ${ }^{1 *}$, PEI WANG ${ }^{2 *}$, DA HUANG $^{1}$, LUMING XU $^{3}$, XIAOBEI WANG ${ }^{1}$, LIN WANG $^{1}$ and LIHUA HU ${ }^{1}$ \\ Departments of ${ }^{1}$ Clinical Laboratory Medicine, ${ }^{2}$ Gastroenterology, and ${ }^{3}$ Regenerative Medicine Center, \\ Union Hospital, Tongji Medical College, Huazhong University of Science and Technology, Wuhan, Hubei 430022, P.R. China
}

Received October 12, 2015; Accepted December 28, 2015

DOI: $10.3892 /$ ijo.2016.3336

\begin{abstract}
Accumulating evidence demonstrates the important roles of microRNAs (miRNAs) in tumor development and progression. miR-26a has been reported to be downregulated in several types of cancers including hepatocellular carcinoma, but the underlying mechanism of how miR-26a is repressed remains largely unknown. In the present study, we performed western blot analysis, qRT-PCR, luciferase reporter assay and chromatin immunoprecipitation assay to investigate the relationship between miR-26a and the enhancer of zest homologue 2 (EZH2). CCK-8 assay and colony formation assay were carried out to explore the effect of miR-26a on HCC cells proliferation. We demonstrated that miR-26a was epigenetically repressed by EZH2-mediated H3K27 trimethylation within the miR-26a promoter. Moreover, we confirmed that EZH2 was also a direct target of miR-26a in HCC cells, thus, creating a double-negative feedback loop. Furthermore, miR-26a restoration increased the expressions of its host genes (CTDSPL and CTDSP2). Overexpression of EZH2 abrogated miR-26a induction of CTDSPL and CTDSP2. Restoring the balance of the double-negative feedback loop by miR-26a overpression or EZH2 silence significantly inhibited HCC cell growth. Overexpression of EZH2 rescued the growth inhibition effect of miR-26a. These findings suggest that an imbalanced double-negative feedback loop between EZH2 and miR-26a exists in HCC cells, which contributes to miR-26a deregulation and regulates tumor cells proliferation.
\end{abstract}

Correspondence to: Professor Lihua Hu and Professor Lin Wang, Department of Clinical Laboratory Medicine, Union Hospital, Tongji Medical College, Huazhong University of Science and Technology, No. 1277 Jie Fang Road, Wuhan, Hubei 430022, P.R. China

E-mail: hulihuaunion@126.com

E-mail: katelinwang@126.com

*Contributed equally

Key words: enhancer of zest homologue 2, miR-26a, hepatocellular carcinoma, histone methylation, proliferation, colony formation

\section{Introduction}

Hepatocellular carcinoma (HCC) represents the second leading cause of cancer-related deaths worldwide (1), resulting in 700,000 deaths per year. Although therapeutic options such as surgical resection and liver transplantation are used to improve the outcomes and decrease mortality of HCC patients, the 5-year survival rate is still less than $30 \%$ (1). In many cases, HCC is detected at advanced stage with limited therapeutic options. Thus, a better understanding of the molecular mechanism underlying HCC carcinogenesis will help to indentify novel diagnostic biomarkers and develop targeted therapies.

MicroRNAs (miRNAs) are small non-coding RNAs with a length of 20-25 nucleotides that post-transcriptionally regulate gene expression by complementary binding to the 3'untranslated region (3'UTR) of target mRNAs, inducing degradation or translational repression (2). miRNAs have been well established to closely associate with carcinogenesis and progression, where they function as oncogenes or tumor suppressors depending on their targets. miR-26a, an early discovered miRNA, comprises miR-26a-1 and miR-26a-2, which are located in the intron of CTD small phosphatase like (CTDSPL) and CTD small phosphatase 2 (CTDSP2), respectively. miR-26a-1 and miR-26a-2 are expressed concomitantly with their host genes in physiological and pathological conditions (3). It has been reported that miR-26a is downregulated in several types of cancers including HCC $(4,5)$. miR-26a suppressed tumor growth and metastasis by targeting multiple oncogenes and cell cycle-related genes such as IL-6 (5), Zcchc11 (6), ITGA5 (7), CCND2 and CCNE2 (8) in HCC. However, the underlying mechanism of how miR-26a is repressed remains largely unknown.

Epigenetic regulation plays a central role in the regulation of genes. Polycomb repressive complex 2 (PRC2) is an important epigenetic regulator that represses tumor suppressor genes such as KFL2, ID4 and E-cadherin by chromatin modification (9-12). The enhancer of zest homologue 2 (EZH2) is the catalytically active subunit of PRC2. It suppresses gene expression by inducing the trimethylation of lysine 27 on histone 3 (H3K27) (13). EZH2 has been shown overexpressed in a variety of cancers including HCC $(14,15)$. EZH2 plays an important role in carcinogenensis, progression and metastasis, where it functions as an oncogene (16-18). Recently, several 
studies reported that miRNAs such as miR-214 (19), miR-101 (20), miR-200s (21-23), miR-218 (24), miR-143 (25), miR-622 (26) and miR-31 (27), could be epigenetically suppressed by EZH2-mediated H3K27 trimethylation. It seems that overexpression of EZH2 may partly explain the suppression of tumor suppressor miRNAs. Thus, we presume that miR-26a could be silenced by EZH2-mediated epigenetic mechanisms. Moreover, EZH2 has been reported to be a direct target of miR-26a in nasopharyngeal (28), lung (29) and prostate cancer (30). However, the regulatory mechanism of EZH2 expression and the relationship between EZH2 and miR-26a in HCC are still unclear.

In the present study, we demonstrate that miR-26a is repressed by EZH2-mediated $\mathrm{H} 3 \mathrm{~K} 27$ trimethylation within the miR-26a promoter and confirm that EZH2 is a direct target of miR-26a in HCC cells. EZH2 and miR-26a form a doublenegative feedback loop, contributing to miR-26a deregulation and regulating $\mathrm{HCC}$ cell growth. Our findings provide a better understanding of HCC pathogenesis.

\section{Materials and methods}

Cell culture and transfection. LO2, HepG2 and SMMC7721 cell lines were obtained from the Cell Bank of Chinese Academy of Sciences (Shanghai, China), and were maintained at $37^{\circ} \mathrm{C}\left(5 \% \mathrm{CO}_{2}\right)$ in Dulbecco's modified Eagle's medium (DMEM) supplemented with $10 \%$ fetal bovine serum (FBS; Gibco, Grand Island, NY, USA). All experiments were performed when cells were at logarithmic growth phase. For transfection, cells were plated in 6-well plates for $24 \mathrm{~h}$ to ensure $70-80 \%$ confluence. Then, the cells were transfected with $4 \mu \mathrm{g}$ pcDNA3.1, pcDNA3.1-miR-26a, pcDNA3.1-EZH2 and sh-EZH2, or $100 \mathrm{nM}$ miR-26a mimics and control mimics using Lipofectamine 2000 reagent (Invitrogen, Carlsbad, CA, USA) according to the manufacturer's instruction. The miR-26a mimics and control mimics were purchased from Guangzhou RiboBio Co., Ltd. (Guangzhou, China).

Construction of expression plasmids. To construct miR-26a expression plasmid (pcDNA3.1-miR-26a), a 254-bp DNA fragment containing pre-miR-26a and the 5'- and 3'-flanking sequence was amplified from human genomic DNA and cloned into the $K p n I$ and $E c o$ RI sites of pcDNA3.1 vector. The primers were 5'-AGGGGTACCGGCTGGGGTCAGAAAT-3' (forward) and 5'-GCAGAATTCGCTACATGCAAAGGGC AG-3' (reverse). The underlined bases represent the restriction enzyme cutting sites. EZH2 expression plasmid (pcDNA3.1EZH2) was generated by cloning the coding sequence of EZH2 without 3'UTR into pcDNA3.1+ vector. For sh-EZH2 plasmid construction, two shRNAs targeting human EZH2 mRNA (shRNA-1 5'-CCCAACATAGATGGACCAAAT-3'; shRNA-2 5'-GAGTATGTTTAGTTCCAATAA-3') were designed and cloned into pGenesil-1 plasmid.

Quantitative real-time PCR. TRIzol reagent (Invitrogen) was used to extract total RNA according to the manufacturer's instructions. The expression of miR-26a was determined by a stem-loop qRT-PCR assay. Reverse transcription of miR-26a and U6 was performed by using PrimeScript ${ }^{\mathrm{TM}}$ RT reagent kit (Takara Bio, Shiga, Japan) with specific RT primer. For mRNA analysis, $1 \mu \mathrm{g}$ of total RNA was reverse-transcribed using PrimeScript $^{\mathrm{TM}}$ RT Master Mix (Takara Bio). Subsequently, real-time PCR was performed using SYBR-Green PCR Master Mix (Takara). All PCR reactions were performed under the conditions of $5 \mathrm{~min}$ at $95^{\circ} \mathrm{C}$, followed by 40 cycles of $5 \mathrm{sec}$ at $95^{\circ} \mathrm{C}, 30 \mathrm{sec}$ at $60^{\circ} \mathrm{C}$ and $30 \mathrm{sec}$ at $72^{\circ} \mathrm{C}$ on a Stratagene Mx3000P system. The relative expression levels of miR-26a and mRNAs were calculated by $2^{-\Delta \Delta \mathrm{Ct}}$ method using GAPDH or U6 as internal control. The primers used for qRT-PCR were as follows: miR-26a, 5'-GTCGTATCCAGTGCAGGGTCC GAGGTATTCGCACTGGATACGACAGCCA-3' (RT), 5'-GC CCGCTTCAAGTAATCCAGG-3' (forward) and 5'-GTGCA GGGTCCGAGGT-3' (reverse); U6, 5'-CTCGCTTCGGCAG CACA-3' (forward) and 5'-AACGCTTCACGAATTTGC GT-3' (reverse); EZH2, 5'-AGTTTGCTGCTGCTCTCAC-3' (forward) and 5'-GTTCTCTCCCCCCGTTTC-3' (reverse); CTDSPL, 5'-TGCTGAGGGAGGGGAGTGAG-3' (forward) and 5'-GCAGCATGCCACAGGTTGTC-3' (reverse); CTDSP2, 5'-TCACCAGGTGTATGTGCTCAA-3' (forward) and 5'-AA GACTCACGGAATAGGCG-3' (reverse); E-cadherin, 5'-CC CCATACCAGAACCTCG-3' (forward) and 5'-TTCTTGGG TTGGGTCGTT-3' (reverse); GAPDH, 5'-GAAGGTGAAG GTCGGAGTC-3' (forward) and 5'-GAAGATGGTGATGG GATTTC-3' (reverse).

Western blot analysis. Cells were lysed using RIPA reagent (Beyotime Institute of Biotechnology, Jiangsu, China). Protein lysates were sonicated and centrifuged at 12,000 rpm for $5 \mathrm{~min}$. Then protein extracts were subjected into $12 \%$ SDS-PAGE and transferred to NC membrane (Milipore, Billerica, MA, USA). After blocked with 5\% non-fat milk, the membrane was incubated with rabbit anti-human specific primary antibodies (anti-EZH2, 1:1,000 and anti-GAPDH, 1:2,000; Cell Signaling Technology, Danvers, MA, USA) at $4^{\circ} \mathrm{C}$ overnight, followed by incubation with HRP-conjugated goat anti-rabbit secondary antibody (1:5,000; Wuhan Boster Biological Technology, Ltd., Wuhan, China) at room temperature for $1 \mathrm{~h}$. The membrane was detected with BeyoECL plus kit (Beyotime Institute of Biotechnology) and the intensity of protein fragments was quantified using Image Lab software (Bio-Rad Laboratories, Hercules, CA, USA). The signals of the detected bands were normalized to GAPDH band, and the values were presented as the ratio between treated cells and control cells.

CCK-8 assay. Cells were seeded in 96-well plates ( $2 \times 10^{3} /$ well) at a final volume of $100 \mu \mathrm{l}$. After transfection, the cells were cultured for another 24, 48, 72 and $96 \mathrm{~h}$. Then, $10 \mu \mathrm{l}$ of CCK-8 reagent (Beyotime Institute of Biotechnology) was added into each plate at different time-points and incubated at $37^{\circ} \mathrm{C}$ for $1 \mathrm{~h}$. The absorbance was measured by a microplate reader at $450 \mathrm{~nm}$.

Colony formation assay. Forty-eight hours after transfection, cells were seeded in 6-well plates (500 cells/well) and maintained in DMEM medium containing 10\% FBS for 2 weeks. The cells were then fixed and stained with $1 \%$ crystal violet. The number of colonies was counted under a microscope (Olympus IX83; Olympus, Tokyo, Japan). All experiments were repeated three times and the values were reported as the ratio between treated cells and control cells. 
Luciferase reporter assay. To construct EZH2-3'UTR-WT plasmid, a 274-bp DNA fragment of EZH2 3'UTR containing the predicted binding site of miR-26a was amplified from human genomic DNA and cloned into a reporter vector as previously described (31). The primers for EZH2-3'UTR-WT were as follows: 5'-ATAGAATTCCATCTGCTACCTCC TCC-3' (forward) and 5'-CGCAAGCTTGATTCAACAAGG AC-3' (reverse). The EZH2-3'UTR-MUT plasmid, which converted the miR-26a binding site 'TACTTGAA' to 'CTGCAGAA', was generated by site-specific mutagenesis based on EZH2-3'UTR-WT plasmid. The primers for EZH23'UTR-MUT plasmid were: 5'-AACTTTGAATAAAGAACT GCAGAACTTGTCCTTGTTG-3' (forward) and 5'-AACAA GGACAAGTTCTGCAGTTCTTTATTCAAAGTTG-3' (reverse).

To investigate the transcriptional regulation of miR-26a, a 2147-bp fragment of CTDSPL/miR-26a-1 promoter (-1521/+626) and a 1569-bp fragment of CTDSP2/miR-26a-2 promoter (-1322/+247) were amplified by PCR and cloned into the pGL3-basic plasmid, generating pGL3-26a-1 and pGL326a-2. The primers for pGL3-26a-1 were as follows: 5'-ACGGGTACCCAGAGTGTGGCCTGCAAGTTGGAA-3' (forward) and 5'-TCAAAGCTTCGCACACACATTCCACA ACCCCTCA-3' (reverse). The primers for pGL3-26a-2 were: 5'-TCACTCGAGATTGGGGTTGGATTTAGC-3' (forward) and 5'-AACAAGCTTCGAAAACAAGAGGAGGAAAT-3' (reverse).

For luciferase reporter assay, HepG2 cells were seeded in 96-well plates until 70-80\% confluence. Then, $50 \mathrm{ng}$ of the constructed EZH2 3'UTR reporter plasmids were cotransfected with either $100 \mathrm{nM}$ miR-26a mimics or control mimics, and $5 \mathrm{ng}$ of pRL-TK plasmid (Promega, Madison, WI, USA); $50 \mathrm{ng}$ of the constructed miR-26a promoter reporter plasmids were cotransfected with $200 \mathrm{ng}$ of either pcDNA3.1-EZH2 or pcDNA3.1 and 5 ng of pRL-TK. The pRL-TK plasmid was used as internal control. Forty-eight hours after transfection, the luciferase activity was measured using the Dual-luciferase reporter assay system (Promega).

Chromatin immunoprecipitation. CHIP assay was performed using EpiQuik ${ }^{\mathrm{TM}}$ chromatin immunopercipitation kit (Epigentek, Farmingdale, NY, USA) according to the manufacturer's protocol. Briefly, the cells were transfected with sh-EZH 2 or sh-control for $48 \mathrm{~h}$ prior to formaldehyde fixation. After cell lysis, the chromatin was fragmented to $200-1,000 \mathrm{bp}$. The DNA fragment was then enriched with anti-EZH2, antiH3K 27me3 or normal IgG antibodies (Cell Signaling Technology). The purified DNA was used as template and quantified by qRT-PCR method. The primers for CHIP were as follows: 26a1s1-F 5'-AGGCTGAGGAGGCACTTTG-3', 26a1s1-R 5'-AGTGGGCATTTTCGGGTG-3', 26a1s2-F 5'-GC CGACTGAGCCAGTGTGA-3', 26a1s2-R 5'-AACTTCTCT GCGGGCGTG-3', 26a2s-F 5'-GAGAGCCAATCGGAGC AC-3', 26a2s-R 5'-TGGGACCCTCCATCTGTG-3'.

Statistical analysis. Data were analyzed using GraphPad Prism 5.0 software (GraphPad Software, Inc., La Jolla, CA, USA). The significance of differences between two groups was evaluated by the Student's two-tail t-test. $\mathrm{P}<0.05$ was considered to indicate a statistically significant result.
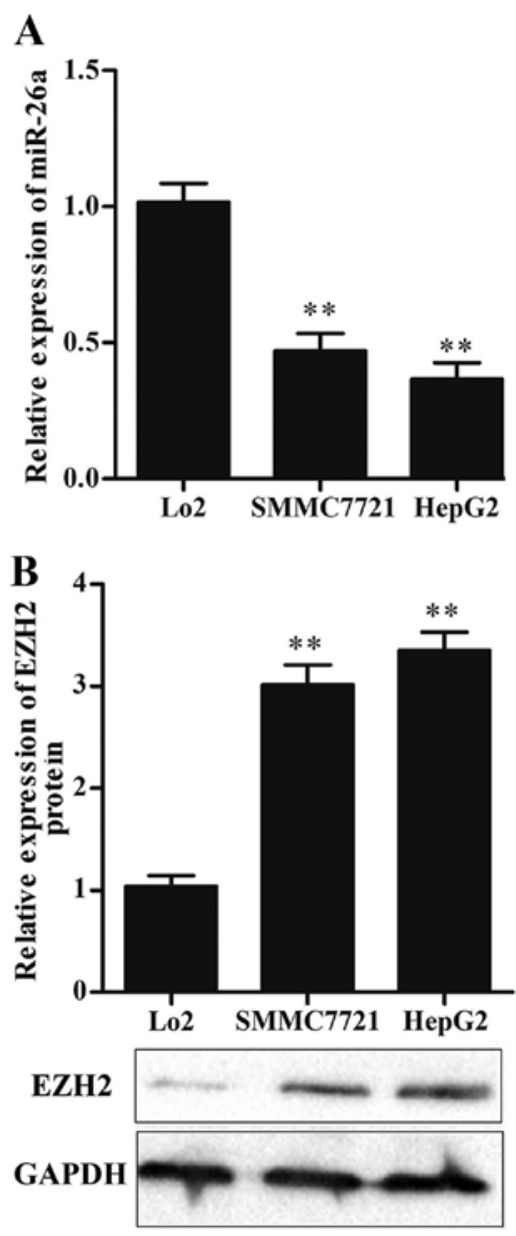

Figure 1. Expression levels of miR-26a and EZH2 in HCC cell lines. (A) The expression of miR-26a in normal liver cell line and HCC cell lines, detected by qRT-PCR. (B) The expression of EZH2 in normal liver cell line and HCC cell lines, detected by western blot analysis. ${ }^{*} \mathrm{P}<0.05$ and ${ }^{* *} \mathrm{P}<0.01$ vs. Lo2

\section{Results}

The expression of miR-26a is downregulated while EZH2 is upregulated in HCC cell lines. To determine the expression level of miR-26a in HCC cells, two HCC cell lines (HepG2 and SMMC7721) and a normal liver cell line (Lo2) were used to detect miR-26a expression levels by qRT-PCR. The result showed that the expression level of miR-26a was significantly decreased in HCC cells compared with that in Lo2 cells $(\mathrm{P}<0.01)$ (Fig. 1A). Moreover, we detected the level of EZH2 by western blot analysis, the result showed that the expression of EZH2 was significantly increased in $\mathrm{HCC}$ cells $(\mathrm{P}<0.01)$ (Fig. 1B).

$m i R-26 a$ is negatively regulated by EZH2. EZH2 as an oncogene, has been reported to epigenetically repress expressions of various tumor suppressor miRNAs such as miR-101, miR-200s and miR-31. To investigate whether miR-26a could be inhibited by EZH2 in HCC cells, we performed loss- and gain-of-function studies. The result showed that knockdown of EZH2 led to a 1.9- and a 1.8-fold increase of miR-26a in HepG2 and SMMC7721 cells, respectively $(\mathrm{P}<0.01)$ (Fig. $2 \mathrm{~A})$. We also detected the expression of miR-26a host genes, CTDSPL 
A

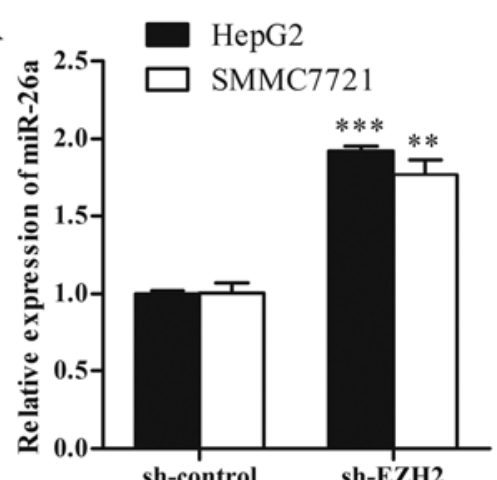

B

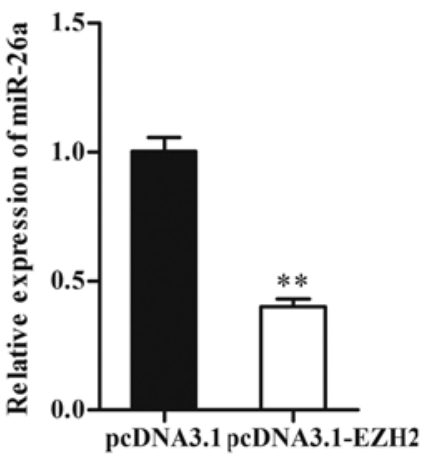

C

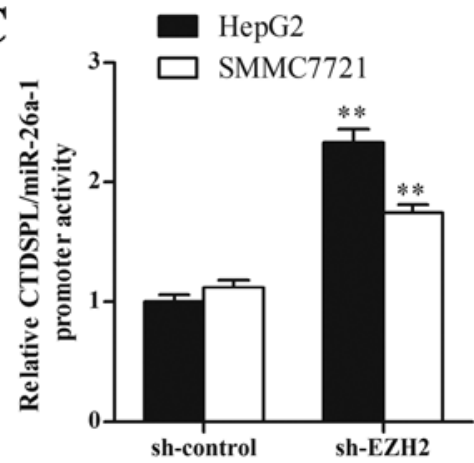

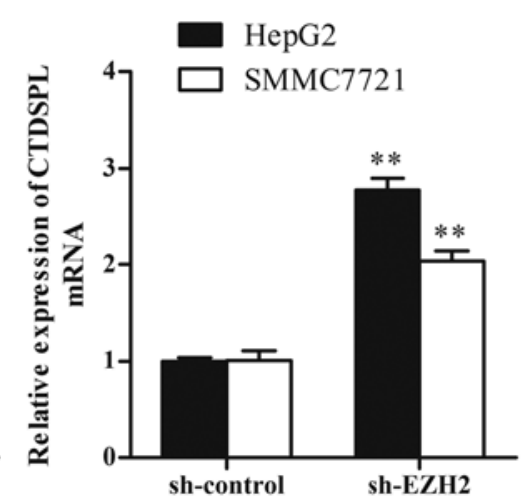
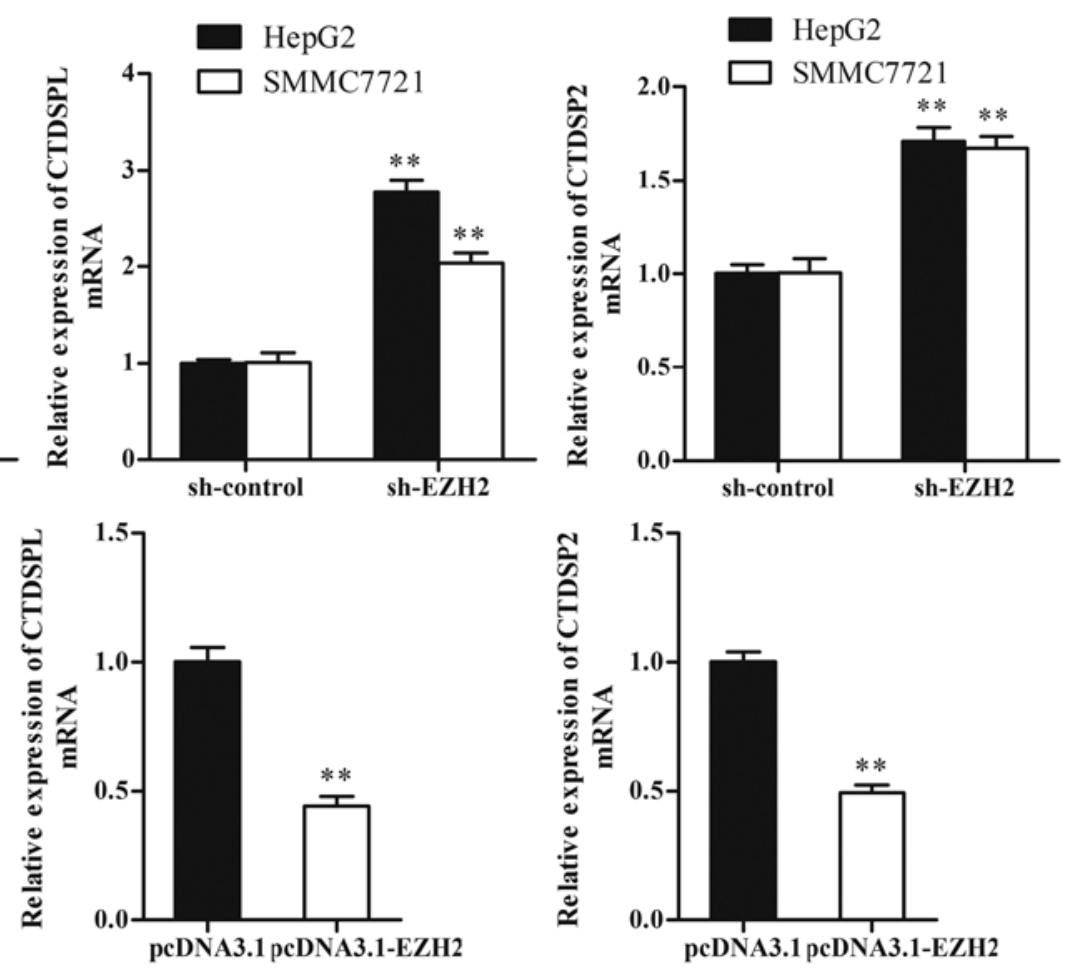

Figure 2. miR-26a is negatively regulated by EZH2. (A) HepG2 and SMMC7721 cells were transfected with sh-EZH2 or sh-control for 48 h, the expression of indicated genes was determined by qRT-PCR. (B) Lo2 cells were transfected with pcDNA3.1-EZH2 or pcDNA3.1. Forty-eight hours after transfection, the expression of indicated genes was determined by qRT-PCR. (C) Knockdown of EZH2 increased miR-26a promoter activity. HepG2 and SMMC7721 cells were cotransfected with pGL3-26a-1 or pGL3-26a-2, and either sh-EZH2 or sh-control. Forty-eight hours after transfection, luciferase activity was measured. ${ }^{* *} \mathrm{P}<0.01$ compared with control.

and CTDSP2, which were reported to be concomitantly expressed with miR-26a. As shown in Fig. 2B, knockdown of EZH2 increased the expressions of CTDSPL and CTDSP2 $(\mathrm{P}<0.01)$. Ectopic expression of EZH2 significantly decreased the expression of miR-26a and its host genes by $\sim 60 \%$ in Lo2 cells $(\mathrm{P}<0.01)$ (Fig. 2B). Futhermore, luciferase reporter assay revealed that transcriptional activities of CTDSPL/miR-26a-1 and CTDSP2/miR-26a-2 increased significantly in sh-EZH2 treated HCC cells compared with sh-control $(\mathrm{P}<0.01)$ (Fig. 2C).

EZH2 regulates $H 3 K 27$ methylation on the miR-26a promoter. To determine whether EZH2 could regulate histone $\mathrm{H} 3 \mathrm{~K} 27$ methylation on the miR-26a promoter, we performed CHIP assay near the transcription start site on CTDSPL/miR-26a-1 promoter and CTDSP2/miR-26a-2 promoter, which containing rich $\mathrm{CpG}$ islands and were highly conserved according to UCSC Genome Browser (Fig. 3A). As shown in Fig. 3B-D, EZH2 and H3K27me3 were enriched at both CTDSPL/
miR-26a-1 and CTDSP2/miR-26a-2 promoters. Knockdown of $\mathrm{EZH} 2$ decreased the binding of $\mathrm{EZH} 2$ and the level of H3K27me3 on CTDSPL/miR-26a-1 and CTDSP2/miR-26a-2 promoters. The result indicates that miR-26a is repressed by EZH2-mediated H3K27 methylation.

EZH2 is a direct target of miR-26a in HCC cells. As EZH2 has been proposed to be a direct target of miR-26a in several types of cancers, it is of interest to investigate whether this relationship also exists in HCC. To investigate whether miR-26a could regulate EZH2 expression in HCC cells, we transfected HepG2 and SMMC7721 cells with miR-26a mimics at different concentrations to upregulate miR-26a expression and then detected EZH2 expression levels. As shown in Fig. 4A and $\mathrm{B}$, ectopic expression of miR-26a significantly decreased both mRNA and protein levels of EZH2 in a dose-dependent manner. At the dose of $100 \mathrm{nM}$, the mRNA and protein levels of EZH2 were decreased by $\sim 50$ and $70 \%$, respectively $(\mathrm{P}<0.01)$. 
$\mathbf{A}_{-2}$
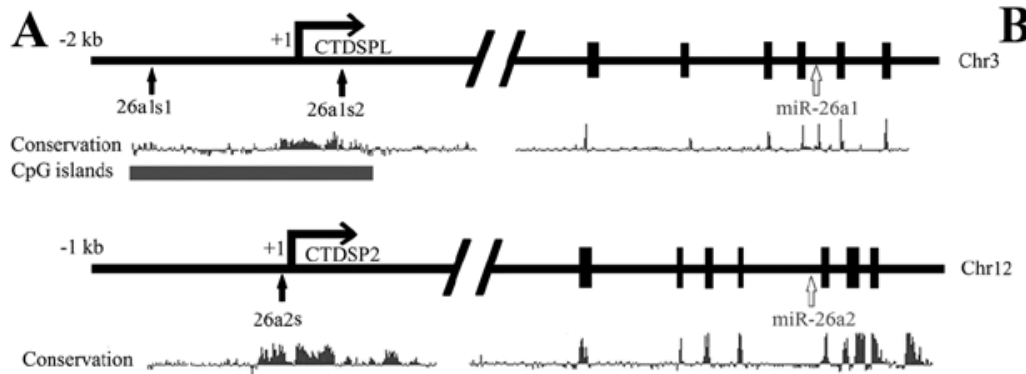
$\mathrm{CpG}$ islands

C

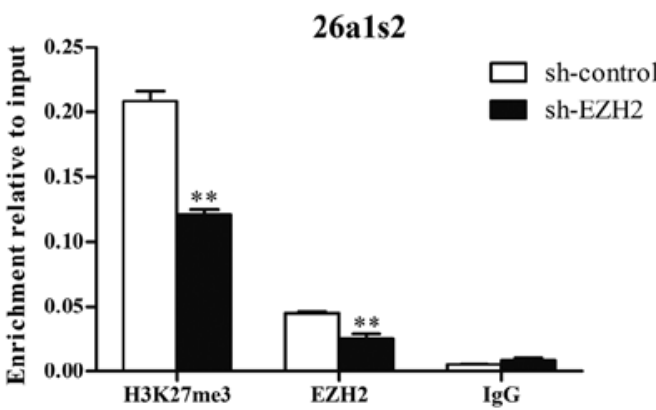

B

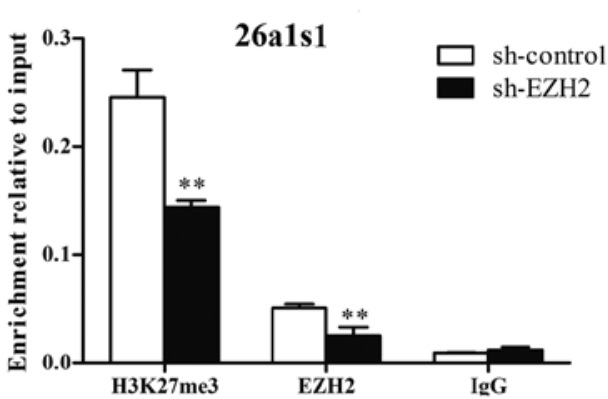

D

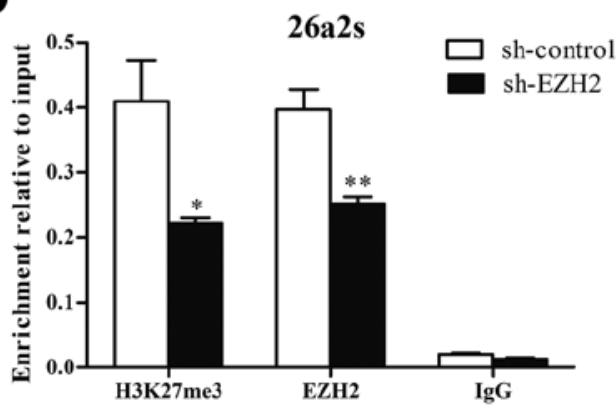

Figure 3. EZH2 regulates H3K27 methylation on the miR-26a promoter. miR-26a and EZH2 form a double-negative feedback loop in HCC cells. (A) Illustration shows the locations of miR-26a-1 and miR-26a-2 within CTDSPL and CTDSP2, and the regions amplified in CHIP-PCR. 26a1s1, 26a1s2 and 26a2s represent the amplified regions. Both CTDSPL/miR-26a-1 and CTDSP2/-miR-26a-2 promoters are highly conserved and CpG-riched. (B-D) CHIP assay shows the enrichment of EZH2 and H3K27me3 on CTDSPL/miR-26a-1 and CTDSP2/-miR-26a-2 promoters. Data are expressed as the fold of enrichment relative to input DNA. ${ }^{\mathrm{P}}<0.05 ;{ }^{* *} \mathrm{P}<0.01$.

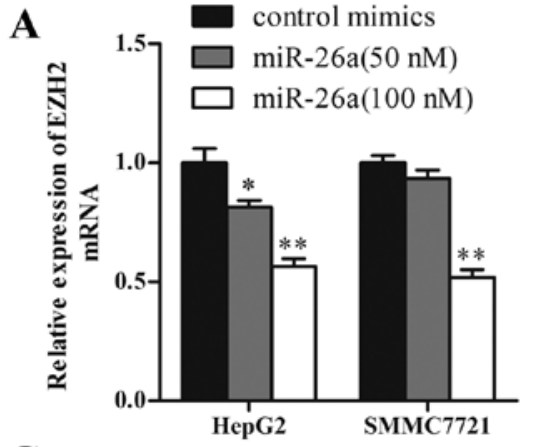

C

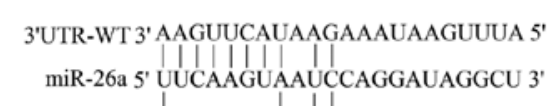
3'UTR-MUT 3' AGACGUCUAAGAAAUAAGUUUA 5'

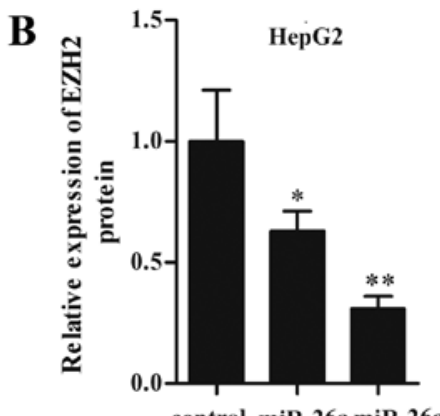

control miR-26a miR-26a mimics $(50 \mathrm{nM})(100 \mathrm{nM})$

EZH2

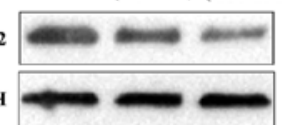

GAPDH

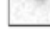

D
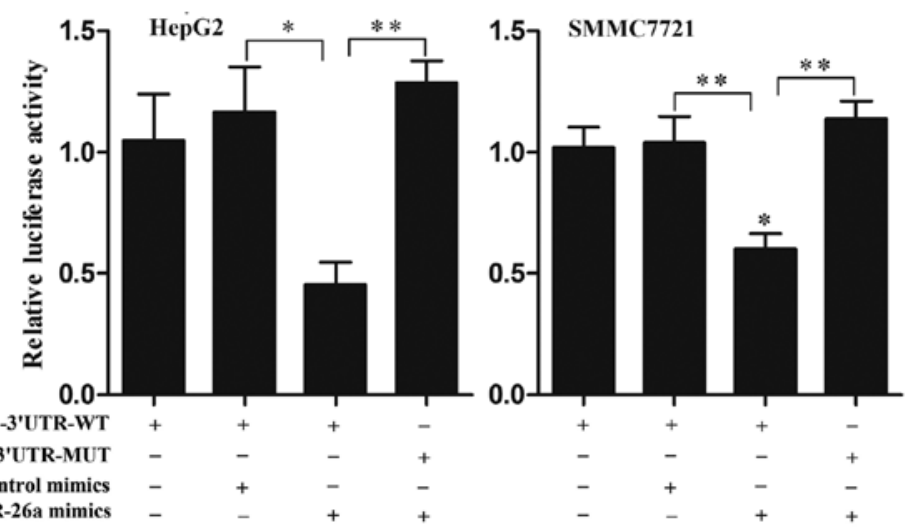

Figure 4. EZH2 is a direct target of miR-26a in HCC cells. (A and B) Supressed expression of EZH2 by miR-26a. (A) qRT-PCR and (B) western blot analysis were used to detect the expression of EZH2 in HCC cells after transfected with different concentrations of miR-26a mimics or control mimics for $48 \mathrm{~h}$. (C) Putative miR-26a binding sequence in the 3'UTR of EZH2 mRNA (www.microrna.org). 3'UTR-MUT was generated by converting the putative miR-26a binding sequence on EZH2 3'UTR to 'CTGCAGAA'. (D) Luciferase reporter assays in HCC cells. HepG2 and SMMC7721 cells were co transfected with pRL-TK, either EZH2-3'UTR-WT or EZH2-3'UTR-MUT, and $100 \mathrm{nM}$ of either miR-26a or control mimics. Forty-eight hours after transfection, luciferase activity was measured. ${ }^{*} \mathrm{P}<0.05 ;{ }^{* *} \mathrm{P}<0.01$. 


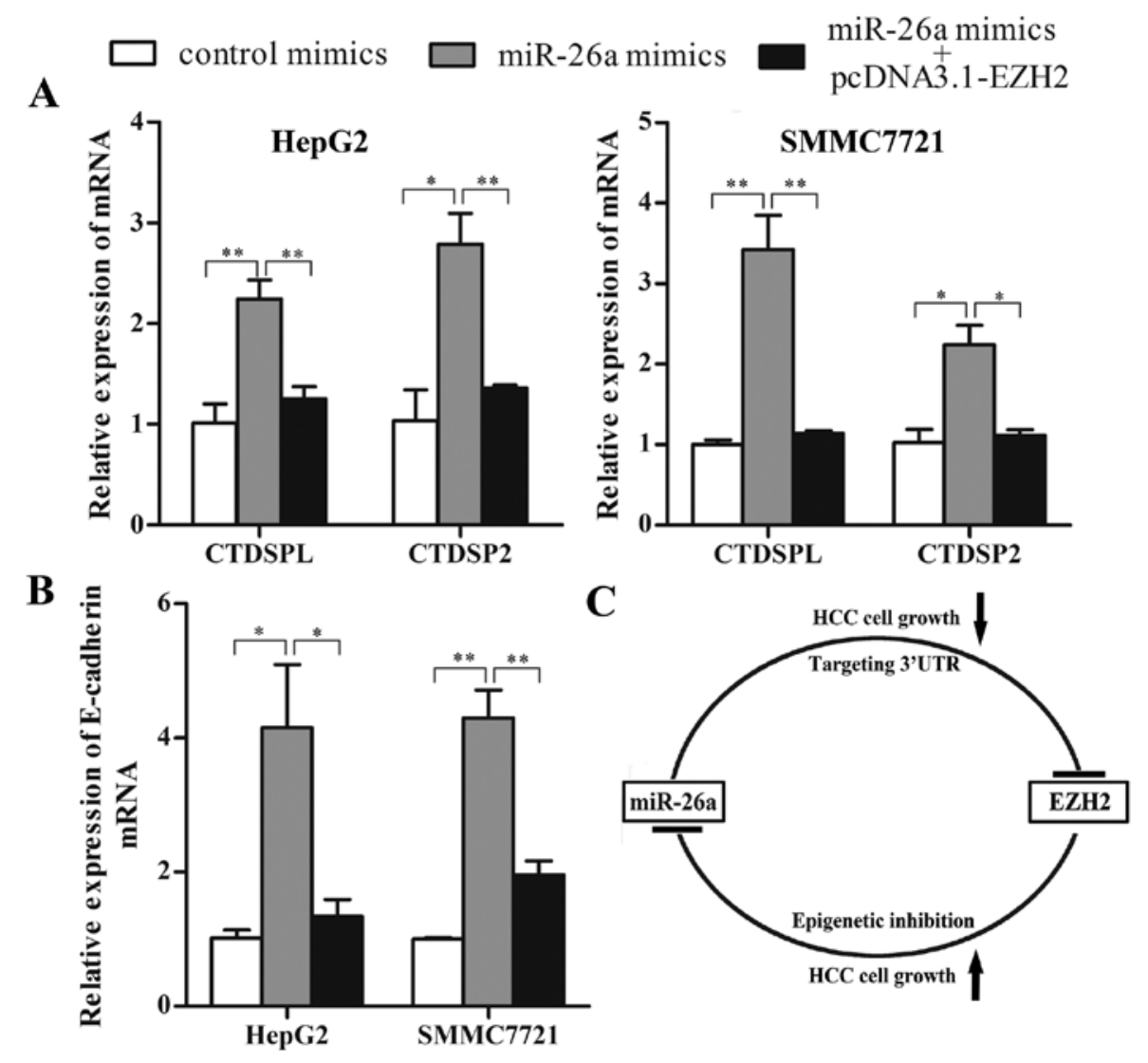

Figure 5. EZH2 and miR-26a form a double-negative feedback loop in HCC cells. (A) Overexpression of EZH2 abrogated the induction of CTDSPL and CTDSP2 expression by exogenous miR-26a. HCC cells were transfected with control mimics or miR-26a mimics, or cotransfected with miR-26a mimics and pcDNA3.1-EZH2 for $48 \mathrm{~h}$. The expression of indicated genes was determined by qRT-PCR. (B) Overexpression of EZH2 abrogated the induction of E-cadherin expression by exogenous miR-26a. (C) Schematic diagram of the double-negative feedback loop formed by miR-26a and EZH2 in HCC cells. ${ }^{* *} \mathrm{P}<0.01$.

Furthermore, luciferase reporter assay was performed to determine whether miR-26a could bind to the 3'UTR of EZH2 mRNA. Luciferase reporter plasmids containing wild-type or mutant EZH2 3'UTR were constructed (Fig. 4C) and cotransfected with miR-26a mimics or control mimics into HepG2 and SMMC7721 cells. As shown in Fig. 4D, miR-26a but not control mimics, significantly decreased the luciferase activity of EZH2-3'UTR-WT reporter, and EZH2-3'UTR-MUT reporter was not affected by miR-26a. These results suggest that EZH2 is a direct target of miR-26a in HCC cells.

miR-26a and EZH2 form a double-negative feedback loop in $H C C$ cells. The above results suggested that EZH 2 and miR-26a may reciprocally regulate each other, forming a double-negative feedback loop. Here, we performed a self-induction experiment of miR-26a. HCC cells were transfected with exogenous miR-26a mimics, and the expression of endogenous miR-26a was detected. Because the detection of endogenous miR-26a could be disturbed by exogenous miR-26a mimics, we detected its host genes (CTDSPL and CTDSP2) instead. As shown in Fig. 5A, compared with control, miR-26a mimics increased the mRNA expression of CTDSPL and CTDSP2 in both HepG2 and SMMC7721 cells. Ectopic expression of EZH2 abrogated miR-26a induction of CTDSPL and CTDSP2. These results benefit the existence of the double-negative feedback loop between EZH2 and miR-26a in HCC. Moreover, we analyzed the expression of E-cadherin, which has been reported to be a target of PRC2. The result showed that miR-26a significantly increased E-cadherin mRNA expression, and the induction was abrogated by EZH2 overexpression (Fig. $5 \mathrm{~B}$ ).

The imbalance of double-negative feedback loop between EZH2 and miR-26a contributes to HCC cell growth. As the double-negative feedback loop between EZH2 and miR-26a is imbalanced in HCC cells, in the present study we restored the balance and investigated the effect of double-negative feedback loop on HCC cell growth. First, we evaluated whether miR-26a restoration could induce growth inhibition of HCC cells, and whether ectopic expression of EZH2 could rescue the growth inhibition effect of miR-26a. miR-26a expression vector (pcDNA3.1-miR-26a) and EZH2 expression vector (pcDNA3.1-EZH2, without 3'UTR) were constructed and confirmed to be effective by qRT-PCR and western blot analysis (Fig. 6A). HepG2 and SMMC7721 cells were transfected with pcDNA3.1 or pcDNA3.1-miR-26a, or cotransfected with pcDNA3.1-miR-26a and pcDNA3.1-EZH2, and then CCK-8 and colony formation assays were performed. As shown in Fig. 6B and C, miR-26a restoration significantly suppressed HCC cell proliferation and colony formation $(\mathrm{P}<0.01)$, and EZH2 abrogated miR-26a-induced cell proliferation inhibition and suppression of colony formation $(\mathrm{P}<0.01)$.

Subsequently, we used the sh-EZH2 vector to suppress EZH2 expression, and investigated whether reduction of EZH2 could mimic the suppressive effect of miR-26a restoration. The 
A
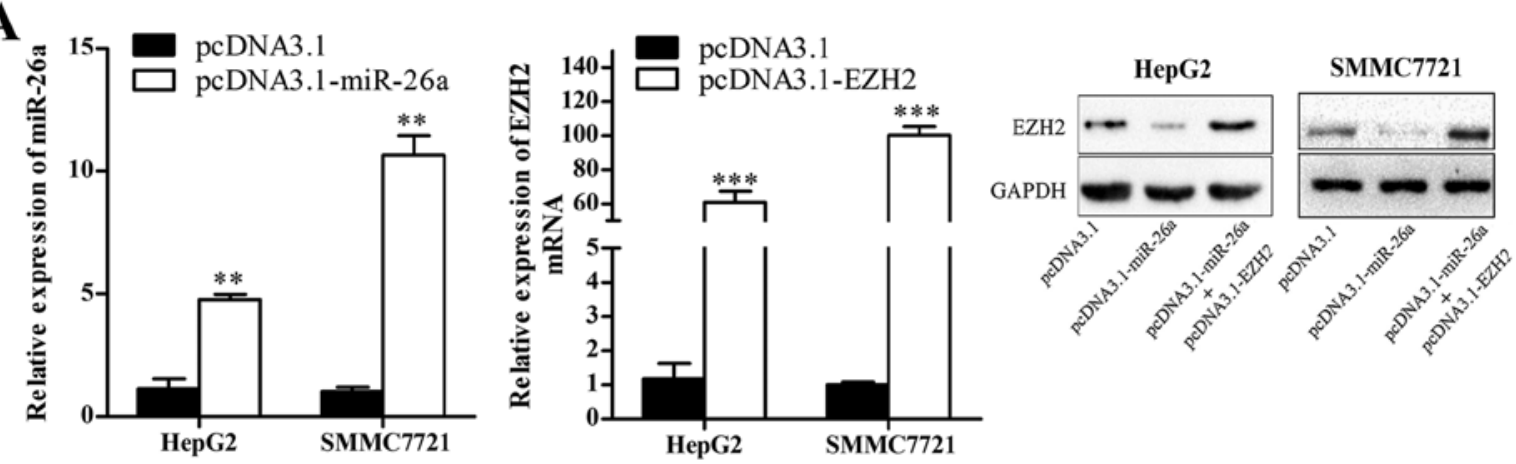

B
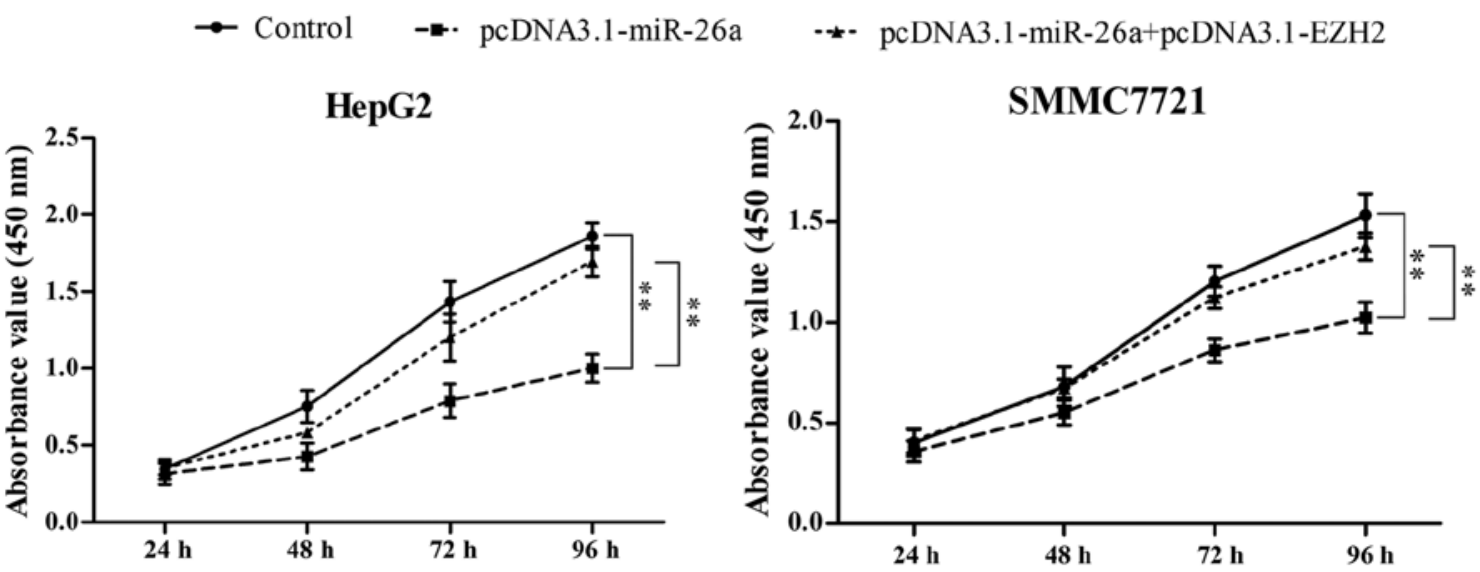

C
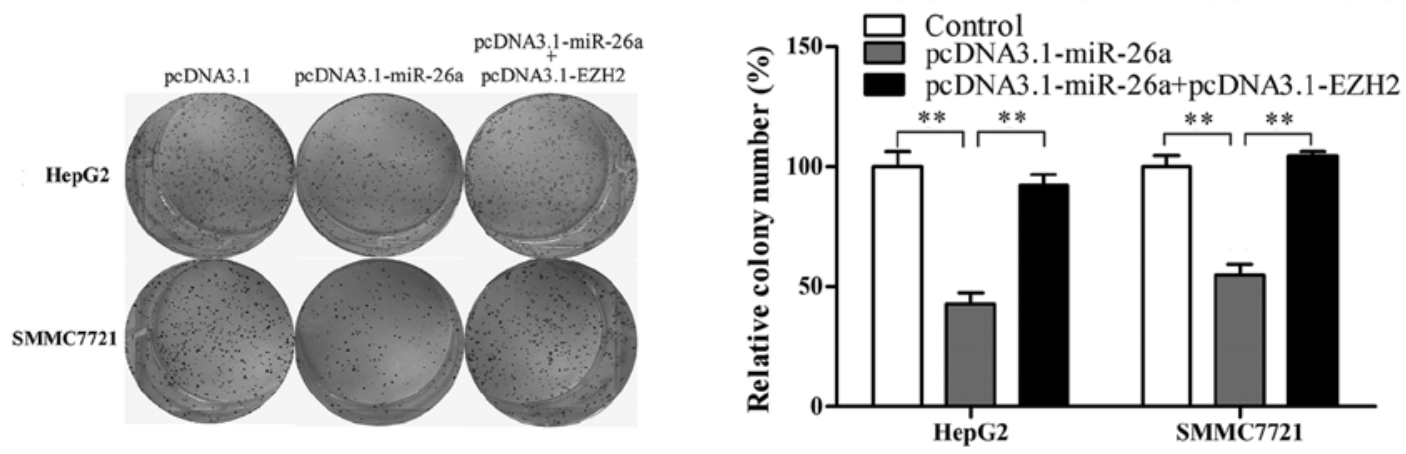

Figure 6. Ectopic expression of EZH2 rescues the growth inhibition effect of miR-26a in HCC cells. (A) Expression levels of miR-26a and EZH2 in HepG2 and SMMC7721 cells after transfected with pcDNA3.1-miR-26a or pcDNA3.1-EZH2 for $48 \mathrm{~h}$. (B) Overexpression of EZH2 abrogates the inhibition effect of miR-26a on HCC cells proliferation. HepG2 and SMMC7721 cells were transfected with pcDNA3.1 or pcDNA3.1-miR-26a, or cotransfected with pcDNA3.1miR-26a and pcDNA3.1-EZH2, and then CCK-8 assays were performed. (C) Overexpression of EZH2 abrogates the inhibition effect of miR-26a on colony formation of HepG2 and SMMC7721 cells. Left, photographs of representative colony formation assays. Right, colonies were analyzed and normalized to the pcDNA3.1 vector. ${ }^{* *} \mathrm{P}<0.01 ;{ }^{* * *} \mathrm{P}<0.001$.

result showed that, similar to miR-26a restoration, knockdown of EZH2 significantly inhibited HCC cell proliferation and colony formation $(\mathrm{P}<0.01)$ (Fig. 7). Taken together, our results suggest that the double-negative feedback loop between EZH2 and miR-26a regulates HCC cell growth.

\section{Discussion}

Accumulating evidence demonstrates the important roles of miRNAs in tumor development and progression (32), but the regulatory mechanisms of miRNA expression are still largely unknown. PRC2 is an epigenetic regulator and plays an important role in gene regulation $(9,10)$. Growing number of studies suggest that, similar to protein coding genes, miRNAs could also be regulated by epigenetic mechanisms
(21,33,34). As the key component of PRC2, EZH2 has been shown to participant in the epigenetic regulation of miRNAs in a variety of cancers. Zhang et al (27) demonstrated that EZH2 suppressed miR-31 expression by regulating H3K27 trimethylation on miR-31 promoter in prostate cancer. Wang et al (20) reported that EZH2 coordinated with c-Myc epigenetically silencing miR-101 expression during hepatocarcinogenesis, showing that EZH2 was a target of miR-101, thus creating a double-negative feedback loop that regulated the process of hepatocarcinogenesis. In the present study, we report that reduction of EZH2 caused a significant increase of miR-26a expression. This effect was accompanied by an increase in transcriptional activity of miR-26a promoter. CHIP assay indicated that EZH2 regulated H3K27 trimethylation on miR-26a promoter. These results are consistent 


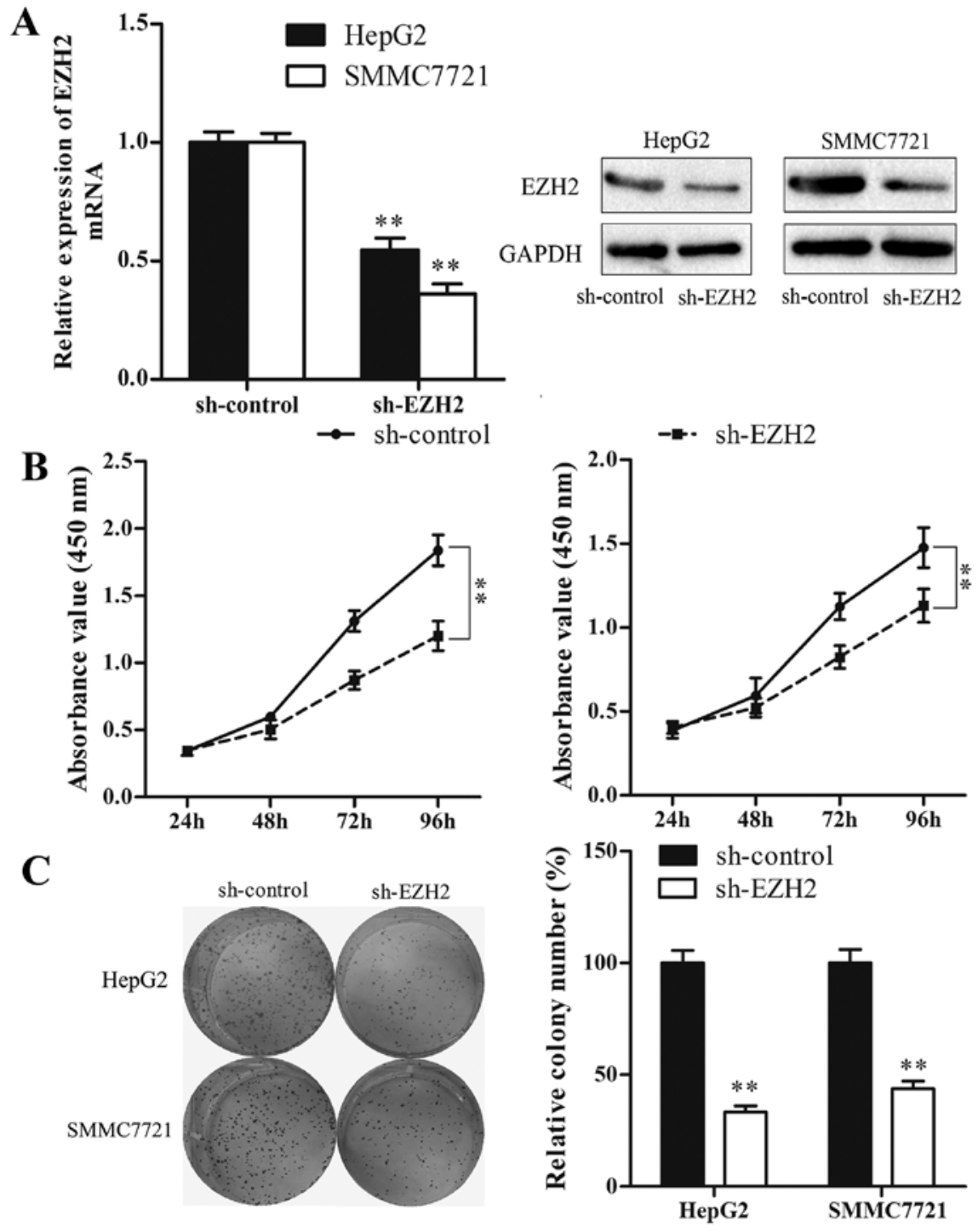

Figure 7. Knockdown of EZH2 inhibits growth in HCC cells. (A) sh-EZH2 effectively reduced mRNA and protein expressions of EZH2 in HCC cells. (B) Knockdown of EZH2 suppressed HCC cell proliferation. HepG2 and SMMC7721 cells were transfected with sh-EZH2 or sh-control, and then CCK-8 assays were performed. (C) Knockdown of EZH2 suppressed colony formation in HCC cells. Left, photographs of representative colony formation assays. Right, colonies were analyzed and normalized to the pcDNA3.1 vector. ${ }^{* *} \mathrm{P}<0.01$ compared with control.

with a previous study by Zhao et al (35), which reported an enrichment of EZH2 on miR-26a promoter, and that reduction of EZH2 increased miR-26a expression in aggressive B-cell lymphomas. Moreover, another study by Borno et al (36) showed that both the expression and the promoter methylation status of miR-26a were associated with EZH2 expression in prostate cancer tissues and cell lines. It seems that epigenetic regulation of miR-26a by EZH2 may be a common mechanism in malignancies.

CTDSPL and CTDSP2, the host genes of miR-26a, have been reported as tumor suppressors. It has been demonstrated that CTDSPL and CTDSP2 dephosphorylated the $\mathrm{ppRb}$ protein and induce cell cycle arrest in HCC (3) and renal carcinoma (37). Moreover, CTDSPL is reported to be downregulated and frequently methylated in non-small cell lung cancer (38), renal cell carcinoma (39) and ovarian cancer (40). Consistently, the bioinformatics analysis revealed that the promoter region of CTDSPL and CTDSP2 contained many $\mathrm{CpG}$ islands. As EZH2 can directly regulate
DNA methylation (41), it is possible that EZH2 suppresses miR-26a by regulating both $\mathrm{H} 3 \mathrm{~K} 27$ trimethylation and DNA methylation.

miR-26a has been previously reported to function as a tumor suppressor in a variety of cancers by targeting multiple oncogenes $(5-8,28-30)$ including EZH2, but its relationship with EZH2 is not fully elucidated in HCC. A recent study by Wang et al (42) reported that miR-26a suppressed EZH2 expression in HepG2 cells. In the present study, we demonstrated that miR-26a suppressed EZH2 expression in a dose-dependent manner, which was consistent with Wang et al (42). We further confirmed that EZH2 was a direct target of miR-26a in HCC cells by luciferase reporter assay. Moreover, we showed that exogenous miR-26a mimics could efficiently induce the endogenous expression of CTDSPL and CTDSP2, and that overexpression of EZH2 disrupted this induction. These data indicate that EZH2 and miR-26a form a double-negative feedback loop, and that overexpression of EZH2 contributes to the imbalance of the double-negative feedback loop, resulting in 
deregulation of miR-26a in HCC. These results also suggest that, besides directly targeting oncogenes, ectopic expression of miR-26a could indirectly upregulate tumor suppressor genes through suppressing EZH2 expression. Thus, miR-26a may function as a master tumor suppressor.

Moreover, we restored the balance of the double-negative feedback loop and found that, similar to miR-26a restoration, knockdown of EZH2 induced cell growth inhibition, and overexpression of $\mathrm{EZH} 2$ rescued the growth inhibition effect of miR-26a in HCC cells. These data suggest that the imbalance of double-negative feedback loop between EZH2 and miR-26a may contribute to $\mathrm{HCC}$ cell growth.

In conclusion, our data suggest that EZH2 and miR-26a reciprocally regulate each other in $\mathrm{HCC}$, forming a doublenegative feedback loop, which contributes to miR-26a deregulation and regulates tumor cell growth. Our findings provide new insights into the regulatory mechanism of miR-26a expression in HCC.

\section{Acknowledgements}

The present study was supported by the National Natural Science Foundation of China (no. 81372259).

\section{References}

1. Maluccio M and Covey A: Recent progress in understanding, diagnosing, and treating hepatocellular carcinoma. CA Cancer J Clin 62: 394-399, 2012.

2. Bartel DP: MicroRNAs: Target recognition and regulatory functions. Cell 136: 215-233, 2009.

3. Zhu Y, Lu Y, Zhang Q, Liu JJ, Li TJ, Yang JR, Zeng C and Zhuang SM: MicroRNA-26a/b and their host genes cooperate to inhibit the G1/S transition by activating the $\mathrm{pRb}$ protein. Nucleic Acids Res 40: 4615-4625, 2012.

4. Kato M, Goto Y, Matsushita R, Kurozumi A, Fukumoto I, Nishikawa R, Sakamoto S, Enokida H, Nakagawa M, Ichikawa T, et al: MicroRNA-26a/b directly regulate La-related protein 1 and inhibit cancer cell invasion in prostate cancer. Int $\mathbf{J}$ Oncol 47: 710-718, 2015

5. Yang X, Liang L, Zhang XF, Jia HL, Qin Y, Zhu XC, Gao XM, Qiao P, Zheng Y, Sheng YY, et al: MicroRNA-26a suppresses tumor growth and metastasis of human hepatocellular carcinoma by targeting interleukin-6-Stat 3 pathway. Hepatology 58: $158-170,2013$

6. Fu X, Meng Z, Liang W, Tian Y, Wang X, Han W, Lou G, Wang X, Lou F, Yen Y, et al: miR-26a enhances miRNA biogenesis by targeting Lin28B and Zcchc11 to suppress tumor growth and metastasis. Oncogene 33: 4296-4306, 2014.

7. Zhang X, Cheng SL, Bian K, Wang L, Zhang X, Yan B, Jia LT, Zhao J, Gammoh N, Yang AG, et al: MicroRNA-26a promotes anoikis in human hepatocellular carcinoma cells by targeting alpha5 integrin. Oncotarget 6: 2277-2289, 2015.

8. Kota J, Chivukula RR, O'Donnell KA, Wentzel EA, Montgomery CL, Hwang HW, Chang TC, Vivekanandan P, Torbenson M, Clark KR, et al: Therapeutic microRNA delivery suppresses tumorigenesis in a murine liver cancer model. Cell 137: 1005-1017, 2009.

9. Sparmann A and van Lohuizen M: Polycomb silencers control cell fate, development and cancer. Nat Rev Cancer 6: 846-856, 2006.

10. Wu Y, Zhang L, Zhang L, Wang Y, Li H, Ren X, Wei F, Yu W, Liu T, Wang X, et al: Long non-coding RNA HOTAIR promotes tumor cell invasion and metastasis by recruiting EZH2 and repressing E-cadherin in oral squamous cell carcinoma. Int $\mathbf{J}$ Oncol 46: 2586-2594, 2015.

11. Chinaranagari S, Sharma P and Chaudhary J: EZH2 dependent $\mathrm{H} 3 \mathrm{~K} 27 \mathrm{me} 3$ is involved in epigenetic silencing of ID4 in prostate cancer. Oncotarget 5: 7172-7182, 2014.
12. Huang MD, Chen WM, Qi FZ, Sun M, Xu TP, Ma P and Shu YQ: Long non-coding RNA TUG1 is up-regulated in hepatocellular carcinoma and promotes cell growth and apoptosis by epigenetically silencing of KLF2. Mol Cancer 14: 165, 2015.

13. Simon JA and Lange CA: Roles of the EZH2 histone methyltransferase in cancer epigenetics. Mutat Res 647: 21-29, 2008.

14. Völkel P, Dupret B, Le Bourhis X and Angrand PO: Diverse involvement of EZH2 in cancer epigenetics. Am J Transl Res 7: 175-193, 2015.

15. Xu L, Beckebaum S, Iacob S, Wu G, Kaiser GM, Radtke A, Liu C, Kabar I, Schmidt HH, Zhang X, et al: MicroRNA-101 inhibits human hepatocellular carcinoma progression through EZH2 downregulation and increased cytostatic drug sensitivity. J Hepatol 60: 590-598, 2014.

16. Gao SB, Zheng QF, Xu B, Pan CB, Li KL, Zhao Y, Zheng QL, Lin X, Xue LX and Jin GH: EZH2 represses target genes through H3K27-dependent and H3K27-independent mechanisms in hepatocellular carcinoma. Mol Cancer Res 12: 1388-1397, 2014.

17. Cheng AS, Lau SS, Chen Y, Kondo Y, Li MS, Feng H, Ching AK, Cheung KF, Wong HK, Tong JH, et al: EZH2-mediated concordant repression of Wnt antagonists promotes $\beta$-catenin-dependent hepatocarcinogenesis. Cancer Res 71: 4028-4039, 2011.

18. Gao SB, Xu B, Ding LH, Zheng QL, Zhang L, Zheng QF, Li SH, Feng ZJ, Wei J, Yin ZY, et al: The functional and mechanistic relatedness of EZH2 and menin in hepatocellular carcinoma. J Hepatol 61: 832-839, 2014.

19. Juan AH, Kumar RM, Marx JG, Young RA and Sartorelli V: Mir-214-dependent regulation of the polycomb protein Ezh2 in skeletal muscle and embryonic stem cells. Mol Cell 36: 61-74, 2009.

20. Wang L, Zhang X, Jia LT, Hu SJ, Zhao J, Yang JD, Wen WH, Wang Z, Wang T, Zhao J, et al: c-Myc-mediated epigenetic silencing of MicroRNA-101 contributes to dysregulation of multiple pathways in hepatocellular carcinoma. Hepatology 59: 1850-1863, 2014.

21. Cao Q, Mani RS, Ateeq B, Dhanasekaran SM, Asangani IA, Prensner JR, Kim JH, Brenner JC, Jing X, Cao X, et al: Coordinated regulation of polycomb group complexes through microRNAs in cancer. Cancer Cell 20: 187-199, 2011.

22. Ning X, Shi Z, Liu X, Zhang A, Han L, Jiang K, Kang C and Zhang Q: DNMT1 and EZH2 mediated methylation silences the microRNA-200b/a/429 gene and promotes tumor progression. Cancer Lett 359: 198-205, 2015.

23. Tao T, Liu D, Liu C, Xu B, Chen S, Yin Y, Ang L, Huang Y, Zhang $\mathrm{X}$ and Chen M: Autoregulatory feedback loop of EZH2/ miR-200c/E2F3 as a driving force for prostate cancer development. Biochim Biophys Acta 1839: 858-865, 2014.

24. Wang B, Liu Y, Luo F, Xu Y, Qin Y, Lu X, Xu W, Shi L, Liu Q and Xiang Q: Epigenetic silencing of microRNA-218 via EZH2-mediated H3K27 trimethylation is involved in malignant transformation of HBE cells induced by cigarette smoke extract. Arch Toxicol: Dec 20, 2014 (Epub ahead of print).

25. Zhang Q, Zhao W, Ye C, Zhuang J, Chang C, Li Y, Huang X, Shen L, Li Y, Cui Y, et al: Honokiol inhibits bladder tumor growth by suppressing EZH2/miR-143 axis. Oncotarget 6: 37335-37348, 2015.

26. Liu H, Liu Y, Liu W, Zhang W and Xu J: EZH2-mediated loss of miR-622 determines CXCR4 activation in hepatocellular carcinoma. Nat Commun 6: 8494, 2015.

27. Zhang Q, Padi SK, Tindall DJ and Guo B: Polycomb protein EZH2 suppresses apoptosis by silencing the proapoptotic miR-31. Cell Death Dis 5: e1486, 2014.

28. Lu J, He ML, Wang L, Chen Y, Liu X, Dong Q, Chen YC, Peng Y, Yao KT, Kung HF, et al: MiR-26a inhibits cell growth and tumorigenesis of nasopharyngeal carcinoma through repression of EZH2. Cancer Res 71: 225-233, 2011.

29. Dang X, Ma A, Yang L, Hu H, Zhu B, Shang D, Chen T and Luo Y: MicroRNA-26a regulates tumorigenic properties of EZH2 in human lung carcinoma cells. Cancer Genet 205: 113-123, 2012.

30. Koh CM, Iwata T, Zheng Q, Bethel C, Yegnasubramanian S and De Marzo AM: Myc enforces overexpression of EZH2 in early prostatic neoplasia via transcriptional and post-transcriptional mechanisms. Oncotarget 2: 669-683, 2011.

31. Li Y, Xie J, Xu X, Wang J, Ao F, Wan Y and Zhu Y: MicroRNA548 down-regulates host antiviral response via direct targeting of IFN- $\lambda 1$. Protein Cell 4: 130-141, 2013.

32. Lin S and Gregory RI: MicroRNA biogenesis pathways in cancer. Nat Rev Cancer 15: 321-333, 2015. 
33. Chen X, He D, Dong XD, Dong F, Wang J, Wang L, Tang J, Hu DN, Yan D and Tu L: MicroRNA-124a is epigenetically regulated and acts as a tumor suppressor by controlling multiple targets in uveal melanoma. Invest Ophthalmol Vis Sci 54: 2248-2256, 2013.

34. Lujambio A, Ropero S, Ballestar E, Fraga MF, Cerrato C, Setién F, Casado S, Suarez-Gauthier A, Sanchez-Cespedes M, Git A, et al: Genetic unmasking of an epigenetically silenced microRNA in human cancer cells. Cancer Res 67: 1424-1429, 2007.

35. Zhao X, Lwin T, Zhang X, Huang A, Wang J, Marquez VE, Chen-Kiang S, Dalton WS, Sotomayor E and Tao J: Disruption of the MYC-miRNA-EZH2 loop to suppress aggressive B-cell lymphoma survival and clonogenicity. Leukemia 27: 2341-2350, 2013.

36. Börno ST, Fischer A, Kerick M, Fälth M, Laible M, Brase JC, Kuner R, Dahl A, Grimm C, Sayanjali B, et al: Genome-wide DNA methylation events in TMPRSS2-ERG fusion-negative prostate cancers implicate an EZH2-dependent mechanism with miR-26a hypermethylation. Cancer Discov 2: 1024-1035, 2012.

37. Kashuba VI, Li J, Wang F, Senchenko VN, Protopopov A, Malyukova A, Kutsenko AS, Kadyrova E, Zabarovska VI, Muravenko OV, et al: RBSP3 (HYA22) is a tumor suppressor gene implicated in major epithelial malignancies. Proc Natl Acad Sci USA 101: 4906-4911, 2004.
38. Senchenko VN, Anedchenko EA, Kondratieva TT, Krasnov GS, Dmitriev AA, Zabarovska VI, Pavlova TV, Kashuba VI, Lerman MI and Zabarovsky ER: Simultaneous down-regulation of tumor suppressor genes RBSP3/CTDSPL, NPRL2/G21 and RASSF1A in primary non-small cell lung cancer. BMC Cancer 10: 75, 2010.

39. Dmitriev AA, Rudenko EE, Kudryavtseva AV, Krasnov GS, Gordiyuk VV, Melnikova NV, Stakhovsky EO, Kononenko OA, Pavlova LS, Kondratieva TT, et al: Epigenetic alterations of chromosome 3 revealed by NotI-microarrays in clear cell renal cell carcinoma. BioMed Res Int 2014: 735292, 2014.

40. Kashuba V, Dmitriev AA, Krasnov GS, Pavlova T, Ignatjev I, Gordiyuk VV, Gerashchenko AV, Braga EA, Yenamandra SP, Lerman M, et al: NotI microarrays: Novel epigenetic markers for early detection and prognosis of high grade serous ovarian cancer. Int J Mol Sci 13: 13352-13377, 2012.

41. Viré E, Brenner C, Deplus R, Blanchon L, Fraga M, Didelot C, Morey L, Van Eynde A, Bernard D, Vanderwinden JM, et al: The Polycomb group protein EZH2 directly controls DNA methylation. Nature 439: 871-874, 2006.

42. Wang G, Sun Y,He Y, Ji C, Hu B and Sun Y: miR-26a promoted by interferon-alpha inhibits hepatocellular carcinoma proliferation and migration by blocking EZH2. Genet Test Mol Biomarkers 19: 30-36, 2015. 